ARTICLE OPEN

Check for updates

\title{
An epitope-based malaria vaccine targeting the junctional region of circumsporozoite protein
}

\author{
Lucie Jelínková ${ }^{1}$, Hugo $\mathrm{Jhun}^{2}$, Allison Eaton ${ }^{2}$, Nikolai Petrovsky $\mathbb{B D}^{3,4}$, Fidel Zavala $\mathbb{D}^{2}$ and Bryce Chackerian ${ }^{1 凶}$
}

A malaria vaccine that elicits long-lasting protection and is suitable for use in endemic areas remains urgently needed. Here, we assessed the immunogenicity and prophylactic efficacy of a vaccine targeting a recently described epitope on the major surface antigen on Plasmodium falciparum sporozoites, circumsporozoite protein (CSP). Using a virus-like particle (VLP)-based vaccine platform technology, we developed a vaccine that targets the junctional region between the $\mathrm{N}$-terminal and central repeat regions of CSP. This region is recognized by monoclonal antibodies, including $\mathrm{mAb} C \mathrm{CI} 43$, that have been shown to potently prevent liver invasion in animal models. We show that CIS43 VLPS elicit high-titer and long-lived anti-CSP antibody responses in mice and is immunogenic in non-human primates. In mice, vaccine immunogenicity was enhanced by using mixed adjuvant formulations. Immunization with CIS43 VLPs conferred partial protection from malaria infection in a mouse model, and passive transfer of serum from immunized macaques also inhibited parasite liver invasion in the mouse infection model. Our findings demonstrate that a $Q \beta$ VLP-based vaccine targeting the CIS43 epitope combined with various adjuvants is highly immunogenic in mice and macaques, elicits long-lasting anti-CSP antibodies, and inhibits parasite infection in a mouse model. Thus, the CIS43 VLP vaccine is a promising pre-erythrocytic malaria vaccine candidate.

npj Vaccines (2021)6:13; https://doi.org/10.1038/s41541-020-00274-4

\section{INTRODUCTION}

Malaria is a major global public health concern, causing 228 million infections and 405,000 deaths worldwide in $2018^{1}$. Although malaria can be caused by several species of the parasitic organism Plasmodium, Plasmodium falciparum is responsible for causing a severe form of the disease with the highest morbidity and mortality, and is one of the leading causes of death in children under 5 years old ${ }^{1}$. Infection is initiated when the female Anopheles mosquito injects sporozoites into the bloodstream of a human host. Sporozoites rapidly migrate to the liver where they transiently multiply within hepatocytes, producing merozoites. Merozoites then enter the bloodstream where they invade erythrocytes, replicate further, and cause the symptoms and pathology of malaria².

Vaccines that target different stages of the malaria life cycle are under development ${ }^{2}$. However, only vaccines that target the preerythrocytic stage have potential for providing sterilizing immunity $^{2}$. One of the primary targets of pre-erythrocytic vaccines is the major $P$. falciparum surface antigen circumsporozoite protein $(P f C S P)^{3,4}$ CSP plays a critical role in facilitating parasite invasion of hepatocytes ${ }^{5}$ and antibodies that target CSP can block infection ${ }^{6,7}$. CSP consists of an immunodominant central repeat region, which contains $\sim 35-38$ copies of an NANP motif and up to four copies of an NVDP motif, flanked by $\mathrm{N}$ - and $\mathrm{C}$-terminal regions ${ }^{8}$. The sole approved malaria vaccine, RTS,S/AS01, is a recombinant proteinbased vaccine developed in 1987, which comprised a large portion of CSP (consisting of 19 NANP repeats and the C-terminal region) fused to the hepatitis $B$ surface antigen $(\mathrm{HBsAg})$ and coexpressed with wild-type (WT) HBsAg to form a particulate antigen, which displays this fragment of CSP at low valency ${ }^{9}$. RTS,S provides only modest protection (30-50\%) from clinical malaria in endemic areas and this protection has been shown to wane rapidly following immunization ${ }^{10-12}$.

Most candidate CSP-targeted vaccines, including RTS,S, have focused on eliciting antibodies against the central repeat region of the protein. Recently, however, potent inhibitory monoclonal antibodies (mAbs), which target a highly conserved epitope within the junctional region between the $\mathrm{N}$-terminal region and the central repeat region have been identified. MGG4, CIS43, and L9 are $\mathrm{mAbs}$ that were isolated from human volunteers immunized with an experimental irradiated whole sporozoite vaccine (PfSPZ); these mAbs are highly effective at inhibiting liver invasion in mouse models of malaria infection ${ }^{13-15}$. These three mAbs bind to overlapping epitopes within the junctional region, a region of CSP that is not included in the RTS,S vaccine ${ }^{4}$. Interestingly, MGG4 and CIS43, but not L9, also appear to be able to weakly bind to the central NANP repeat region, suggesting the possibility that this binding promiscuity contributes to the potent neutralizing activity of this class of antibodies ${ }^{16}$. Although the mechanism of neutralization by these mAbs is not entirely clear, CIS43 has been shown to inhibit a proteolytic cleavage event in CSP that is critical for hepatocyte adhesion and, subsequently, invasion ${ }^{14}$. In addition, L9 and CIS43 have been shown, using mouse infection models, to block hepatocyte infection by preventing parasite egress from sinusoids in the liver and to induce cytotoxic death of sporozoites ${ }^{15}$.

The discovery of a novel site of vulnerability within CSP has several clinical implications. First, neutralizing mAbs could be used as prophylactic treatment in humans traveling to malaria endemic areas. CIS43, e.g., has recently entered Phase I trials for prophylactic prevention of clinical malaria in humans ${ }^{17}$. Second, these data suggest that the junctional region of CSP is a promising target for vaccine development. However, no effective vaccines

\footnotetext{
${ }^{1}$ Department of Molecular Genetics and Microbiology, University of New Mexico School of Medicine, Albuquerque, New Mexico, USA. ${ }^{2}$ W. Harry Feinstone Department of Molecular Microbiology and Immunology, Malaria Research Institute, Johns Hopkins Bloomberg School of Public Health, Baltimore, MD, USA. ${ }^{3}$ Vaxine Pty Ltd, 11 Walkley Avenue, Warradale, Adelaide, SA 5046, Australia. ${ }^{4}$ College of Medicine and Public Health, Flinders University, Adelaide, SA 5042, Australia. ${ }^{凶}$ email: bchackerian@salud.unm.edu
} 
a

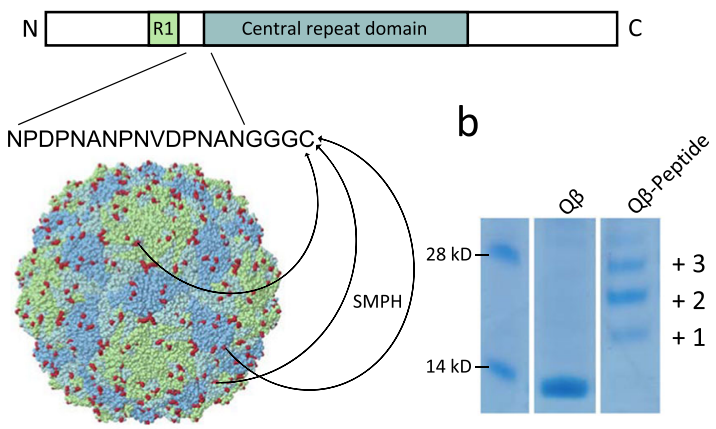

C

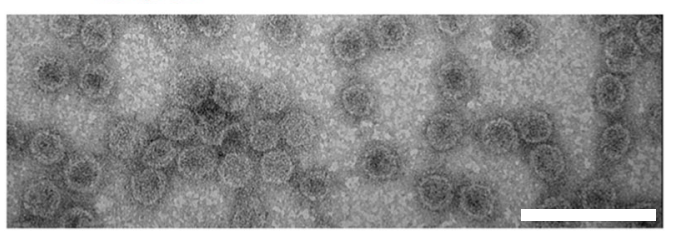

d

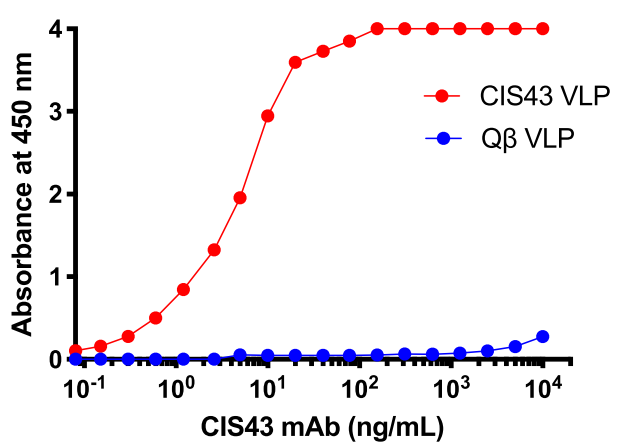

Fig. 1 Characterization of CIS43 VLPs. a Schematic representation of CSP showing the location of the CIS43 epitope and the process of CIS43 VLP conjugation. A 15-amino acid peptide representing the CIS43 mAb epitope was synthesized to include a (Glycine) ${ }_{3}$-Cysteine linker and conjugated to surface-exposed lysine residues (shown in red) on the coat protein of $Q \beta$ bacteriophage VLPs using the bifunctional crosslinker SMPH. $\mathbf{b}$ SDS-PAGE analysis of unconjugated (center lane) or CIS43 peptide conjugated (right lane) Q $\beta$ VLPs. The ladder of bands in the CIS43 VLP lane reflect individual copies of coat protein modified with 1,2, or more copies of the CIS43 peptide. Gel images are derived from the same experiment and were processed in parallel. Size markers are shown in the left lane. The unmodified gel is shown in Supplementary Fig. 2. c Transmission electron micrograph of the CIS43 VLPs. VLPs are visualized at a magnification of 200,000x. Scale bar (in white) represents $100 \mathrm{~nm}$. d Binding of the CIS43 mAb to CIS43 VLPs (red) or wild-type (unmodified) Q $\beta$ VLPs (blue) as measured by ELISA.

targeting this region have been reported in the literature. Tan and colleagues immunized mice with a vaccine consisting of a 19 amino acid peptide encompassing the MGG4 epitope conjugated to keyhole limpet hemocyanin, but the antibodies elicited by this vaccine failed to block sporozoite invasion of hepatocytes ${ }^{13}$. More broadly, there are significant challenges to developing an effective liver-stage vaccine targeting malaria sporozoites. Sporozoites can reach hepatocytes in less than an hour following infection ${ }^{18}$, limiting the window of time in which immune responses can act. In addition, a single infected hepatocyte can seed the blood stage of the malaria life cycle, meaning that effective immunity likely must be sterilizing ${ }^{2}$. Thus, there is a high barrier for vaccinemediated protection-an effective vaccine must elicit very high levels of circulating antibodies and these antibodies must be longlived $^{19}$.

As a potential solution, we investigated the effectiveness of an adjuvanted virus-like particle (VLP)-based vaccine targeting the CIS43 epitope. VLPs are non-infectious, self-assembling particles that are derived from viral structural proteins that can be used as standalone vaccines but also can be applied as platforms for vaccine development ${ }^{20,21}$. VLP-based vaccine design exploits the intrinsic ability of viral structural proteins to self-assemble into highly immunogenic, multivalent particles. These multivalent structures are effective at stimulating strong antibody responses by promoting B-cell receptor crosslinking, leading to robust and long-lasting antibody responses against diverse target antigens ${ }^{20-22}$. In addition, adjuvants play a key role in maximizing the ability of vaccines to induce high titer, high avidity antibodies that are durable over time. In the past, most human malaria vaccine candidates have incorporated either aluminium salts (alum) or oil emulsion adjuvants such as Montanide. However, the former have, by and large, proved insufficiently immunogenic ${ }^{23}$, whereas the latter have suffered from high reactogenicity ${ }^{24}$, raising safety concerns. In recent years, a variety of new adjuvants have become available, raising the possibility to use these to improve malaria vaccine efficacy without compromising safety. Advax adjuvants are proprietary adjuvant formulations produced by Vaxine Pty Ltd, Australia, which include novel adjuvants based on particles including those made from inulin, a plant-based polysaccharide $^{25,26}$. These particulate adjuvants have been shown to be potent enhancers of both cellular and humoral immunity. Advax adjuvants are co-formulated with a range of immune modulators such as TLR9-active CpG oligonucleotides to achieve synergistic adjuvant effects and help steer the immune response in any desired direction.

Here we describe the development and characterization of a bacteriophage VLP-based vaccine targeting the CIS43 epitope. CIS43 VLPs elicit high-titer antibody responses against $P$. falciparum CSP in both mice and macaques, particularly in combination with Advax adjuvants, these antibody responses are highly durable, and vaccination inhibits malaria invasion of the liver in a mouse model.

\section{RESULTS}

\section{Construction and antigenicity of CIS43 VLPs}

The CIS43 mAb epitope was mapped to a 15-amino acid peptide at the $\mathrm{N}$ terminus of the repeat region of CSP (shown schematically in Fig. 1a and in more detail in Supplementary Fig. 1), spanning CSP amino acids $101-115^{14}$. To assess whether a vaccine targeting this epitope could elicit antibodies with CIS43like activity, we engineered RNA bacteriophage VLPs to display the CIS43 epitope at high valency. A peptide representing CSP $_{101-115}$ was synthesized to contain a short Gly-Gly-Gly-Cys linker sequence and then conjugated to the surface lysines on $\mathrm{Q} \beta$ bacteriophage VLPs using a bifunctional crosslinker (shown schematically in Fig. 1a) to produce CIS43 VLPs. Conjugation efficiency was measured by SDS-polyacrylamide gel electrophoresis analysis. Successful peptide conjugation is indicated by an increase in the molecular weight of $Q \beta$ coat protein subunits, reflecting conjugation of one or more peptides to $\mathrm{Q} \beta$ coat protein (Fig. 1b, right lane; the unmodified gel is shown in Supplementary Fig. 2). More than half of all coat protein bound two or more copies of peptide, suggesting that the particles are decorated with the peptide in a dense and multivalent fashion. We estimate that an average of 360 copies of the peptide were conjugated to each Q $\beta$ VLP. CIS43 VLPs were visualized by transmission electron microscopy (TEM), confirming their particulate, multivalent morphology (Fig. 1c). To assess the antigenicity of the CIS43 VLPs, we measured the binding of CIS43 mAb to CIS43 VLPs by enzyme-linked immunosorbent assay (ELISA). As shown in Fig. 1d, CIS43 VLPs were robustly recognized by the CIS43 mAb. These data suggest that the CIS43 epitope peptide is displayed on the $Q \beta$ particles in a manner that emulates its antigenic conformation on CSP. 


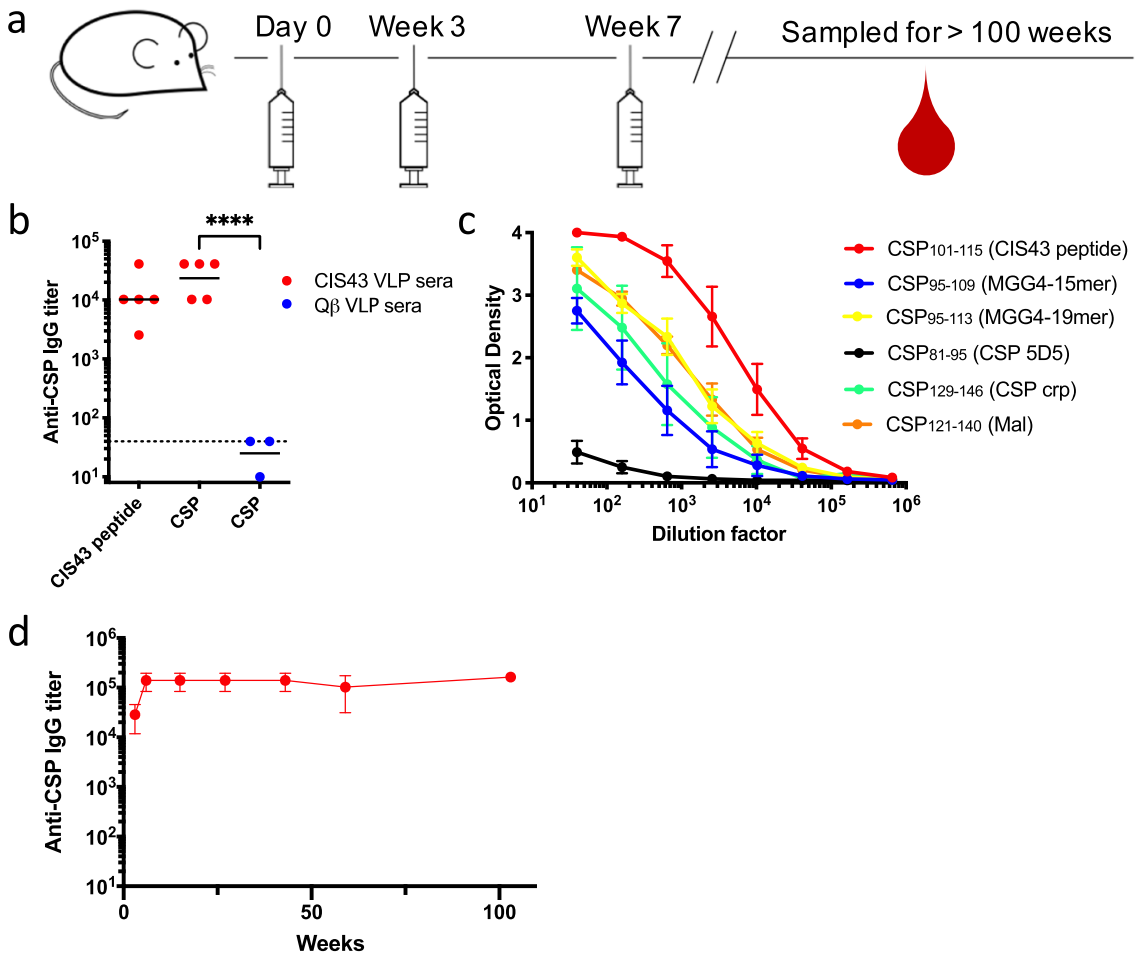

Fig. 2 CIS43 VLPs elicit high-titer and long-lasting antibody responses against CSP. a CIS43 VLP immunization scheme. b lgG titers against CIS43 peptide or CSP were assessed after two immunizations ( 6 weeks after the initial vaccination). Balb/c mice were immunized with $5 \mu \mathrm{g}$ of CIS43 VLPs ( $n=5$; red symbols) or $5 \mu \mathrm{g}$ of WT Q $\beta$ VLPs $(n=3$; blue symbols). Each data point represents an individual mouse and lines represent the geometric mean titers of each group. The dashed line indicates the limit of detection of the ELISA. Statistical significance between groups was determined by $t$-test $\left({ }^{* * *} p<0.0001\right)$. c Sera from CIS43 VLP-immunized Balb/c mice were tested for binding to different CSP peptides (listed in Supplementary Fig. 1) by ELISA. Data represents the mean values from five mice immunized twice with unadjuvanted CIS43 VLPs. Error bars represent SEM. d Geometric mean IgG titers were determined longitudinally for over 100 weeks after the initial vaccination. It is noteworthy that three of the mice in the CIS43 VLP-vaccinated group were killed or died (at weeks 56, 60, and 60) due to health complications unrelated to vaccination and prior to the completion of the study.

\section{CIS43 VLPs induce high-titer antibody responses against CSP}

We have previously shown that multivalent display of peptides on VLPs can stimulate high titer antibody responses. To assess the immunogenicity of the CIS43 VLPs in mice, Balb/c mice were intramuscularly immunized with CIS43 VLPs or, as a negative control, WT Q $\beta$ VLPs and boosted at 3 and 7 weeks (Fig. 2a). Three weeks following the final boost, antibody responses against the CIS43 peptide and full-length recombinant $P$. falciparum CSP were measured by end-point dilution ELISA. Whereas negative control sera collected from mice immunized with WT Q $\beta$ VLPs did not show reactivity to the CIS43 peptide or full-length CSP, CIS43 VLPs elicited strong anti-peptide (Fig. 2b, c) and anti-CSP antibody responses (Fig. 2b). The antibody titer to CSP was slightly higher than the anti-CIS43 peptide titer, possibly because a portion of the CIS43 epitope shares homology to the CSP repeat region ${ }^{14}$. Similar antibody titers were observed in C57BL/6 mice immunized with CIS43 VLPs (Supplementary Fig. 3). To demonstrate that CIS43 VLP-elicited antibodies target the same epitope as the CIS43 mAb, we tested whether sera is able to block the binding of CIS43 mAb to CSP by competition ELISA. We show that sera collected from mice immunized with CIS43 VLPs block CIS43 $\mathrm{mAb}$ binding (Supplementary Fig. 4). Lastly, to interrogate the binding promiscuity of elicited anti-CSP antibodies from immunized Balb/c mice, we tested the ability of sera to bind to several peptides representing epitopes within and flanking the junctional region of CSP (shown in Supplementary Fig. 1) by ELISA (these epitopes are described in refs $13,14,27,28$ ). Although antisera bound most strongly to the peptide corresponding to the CIS43 epitope, we also detected cross-reactive binding to several other CSPderived peptides (Fig. 2c), particularly those peptides that contained NPNV motifs, which are found in the junctional region, but also at the $\mathrm{N}$ terminus of the CSP central repeat region.

We and others have previously shown that vaccination with VLPs can elicit differentiation of long-lived plasma cells (LLPCs), resulting in stable, long-lasting antibody levels ${ }^{29-31}$. It is likely that long-lived circulating antibodies will be required for sustained protection from malaria infection in endemic areas, where reinfection is common. We examined the longevity of the antibody response to CIS43 VLPs in mice. Serum was collected regularly for two years after the third immunization and anti-CSP IgG titers were determined by ELISA. Remarkably, anti-CSP IgG titers were stable over this period, nearly spanning the lifetime of the mice (Fig. 2d). Together, these experiments illustrate that CIS43 VLPs elicit robust and long-lived antibody responses.

\section{Immunization with CIS43 VLPs protects mice from intravenous challenge with Plasmodium}

To examine whether immunization with CIS43 VLPs confers protection from infection, we took advantage of a wellcharacterized mouse infection model for testing CSP-targeted vaccines. In this model, mice are challenged with transgenic Plasmodium berghei $(\mathrm{Pb})$ that have been engineered to express full-length PfCSP (in place of $\mathrm{PbCSP}$ ) and a luciferase reporter $(\mathrm{Pb}$ $P f C S P-L U C)^{32}$. Forty-two hours after infection, liver-stage parasite burden can be quantitated by measuring luciferase levels. Mice were immunized three times with CIS43 VLPs or, as a negative control, WT $\mathrm{Q} \beta$ VLPs, and then were challenged by intravenous injection of $1000 \mathrm{~Pb}$-PfCSP-Luc sporozoites four weeks following the final vaccine boost (Fig. 3a). Liver luciferase levels were compared to unimmunized (naive) mice. Mice immunized with 


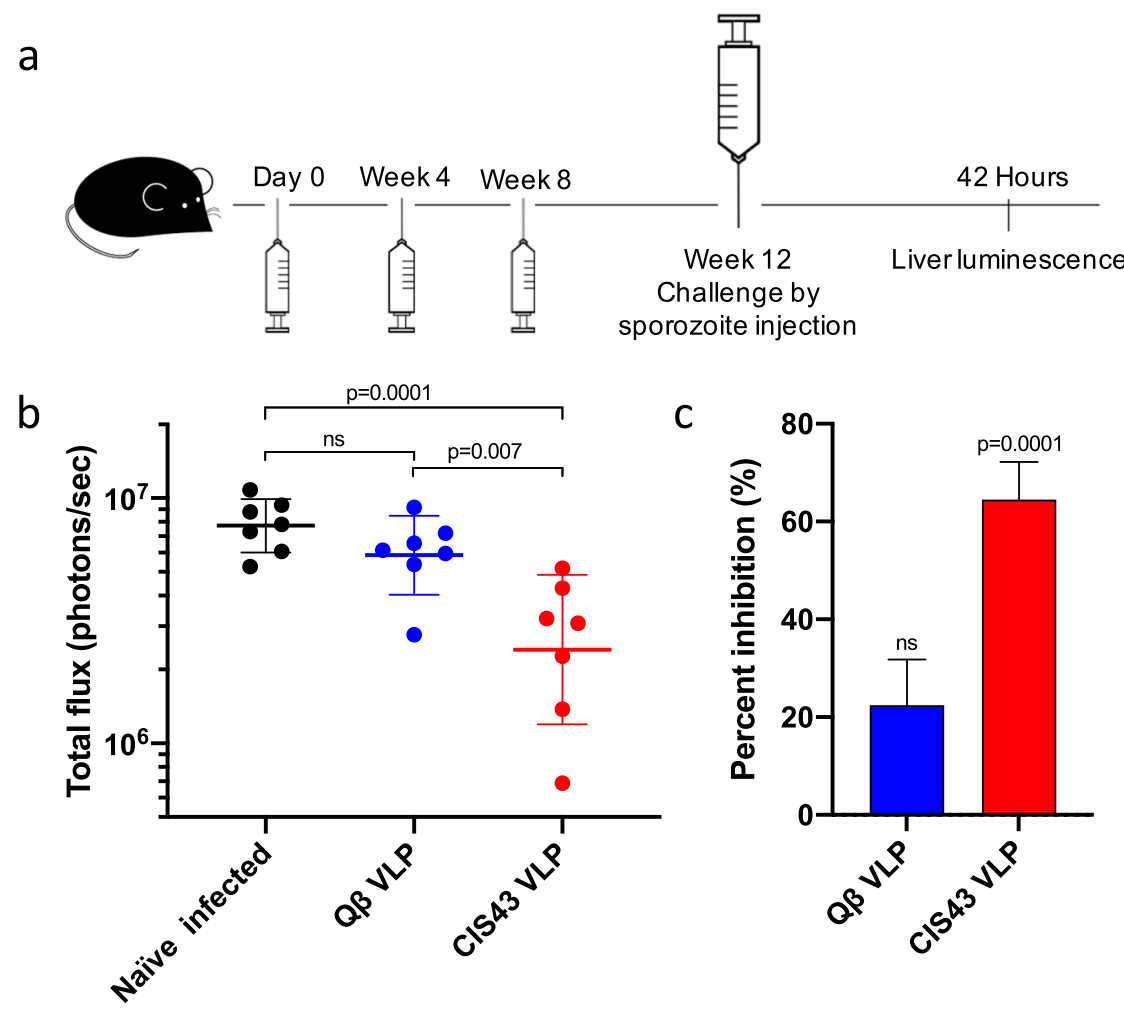

Fig. 3 Immunization with CIS43 VLPs without exogenous adjuvant confers partial protection from Plasmodium infection. a Immunization and challenge scheme. b Parasite liver load (as measured by luminescence) in vaccinated or naive C57BL/6 mice following challenge by sporozoite injection. Horizontal lines represent the geometric mean luminescence of each group, error bars represent SD of the geometric mean. Baseline luminescence was measured at $10^{5}$ photons/s. Groups were compared statistically using one-way ANOVA followed by Dunnett's multiple comparison test. ns; not significant. c Percent inhibition of liver infection, as calculated from luminescence data shown in b. Inhibition of infection is calculated relative to the mean signal in the naive, challenged group of mice. Mann-Whitney test was used to statistically compare each group to the naive infected control group. Error bars represent SEM.

CIS43 VLPs had a significantly reduced ( 64\%) liver-stage parasite burden compared to naive mice (raw data from individual mice is shown in Fig. 3b, mean inhibition data is shown in Fig. 3c). Mice immunized with WT Q $\beta$ VLPs had slightly lower (20\%) parasite burden in the liver compared to naive controls, but this difference was not statistically significant (Fig. 3b, c). These data suggest that immunization with unadjuvanted CIS43 VLPs provides partial inhibition of parasite liver invasion and protection from malaria infection in a rigorous challenge mouse model.

\section{Advax adjuvants increase the immunogenicity of CIS43 VLPs} Immunization with CIS43 VLPs provided partial protection from in vivo challenge. Previous research showed that the protection conferred by passive antibody transfer of CIS43 mAb is dose dependent ${ }^{14,15}$, suggesting that higher antibody levels could potentially provide stronger protection. As a strategy to increase antibody titers elicited by CIS43 VLPs, we tested the compatibility of CIS43 VLPs with a variety of combination adjuvants. These included delta inulin polysaccharides, that have been shown to enhance both the cellular and humoral immune responses to a variety of antigens ${ }^{25,26}$, TLR agonists that induce strongly Th1biased responses, and aluminum salts that induce strong Th2biased responses. Mice were immunized three times with CIS43 VLPs in combination with either delta inulin adjuvant (Advax-1), various combination adjuvant formulations (Advax-2-5), CpG oligonucleotides alone, or without exogenous adjuvant, and then antibody levels were determined by ELISA. As shown in Supplementary Fig. 5, combination adjuvants, including those containing Toll Like Receptor (TLR) 9 or TLR7/8 agonists, increased anti-CSP antibody titers relative to unadjuvanted CIS43 VLPs or
CIS43 VLPs combined with single adjuvants. All of the vaccine/ adjuvant formulations induced a mean $\operatorname{lgG} 1 / \operatorname{lgG} 2$ a ratio of $\sim 5: 1$, a ratio that was statistically indistinguishable from unadjuvanted CIS43 VLPs (Supplementary Fig. 6), and which is consistent with the previous observation that delta inulin acts by enhancing immune activation signals contained within the antigen itself ${ }^{33}$.

Among the adjuvants that were tested, two of the combination adjuvants, Advax-3 (a more Th2-biased co-formulation of CpG55.2 oligonucleotide with aluminum hydroxide) and Advax-4 (a more Th1-biased co-formulation of delta inulin plus a TLR7/8 agonist), had the greatest boosting effect on anti-CSP titers (Supplementary Fig. 4). To more carefully measure the impact of adjuvants, we quantitated anti-CSP antibody concentrations in vaccinated mice by linear regression analysis by CSP ELISA using a standard curve generated using the mouse anti-CSP mAb $2 \mathrm{~A}^{3} 0^{34}$. After two immunizations, mice immunized with unadjuvanted CIS43 VLPs generated a mean anti-CSP IgG level of $22 \mu \mathrm{g} / \mathrm{mL}$. Formulation of CIS43 VLPs with Advax-3 or Advax-4 significantly boosted anti-CSP IgG concentrations by 8.4-fold and 3.8-fold, respectively (Fig. 4a).

To evaluate the longevity of the response, we followed anti-CSP $\lg$ responses over time. As is shown in Fig. 4b, anti-CSP antibody responses declined after the second immunization in all groups, but were restored to peak antibody levels after a third immunization, 18 weeks after the primary immunization. Antibody responses induced by unadjuvanted CIS43 VLPs were remarkably stable after the third immunization, similar to the longevity data shown in Fig. 2c. However, antibody concentrations in mice immunized with CIS43 VLPs with combination adjuvants, particularly with Advax-3, declined following the second immunization, suggesting that some of this increase in total anti-CSP antibody levels may be mediated by differentiation of $B$ cells into short-lived plasma cells 

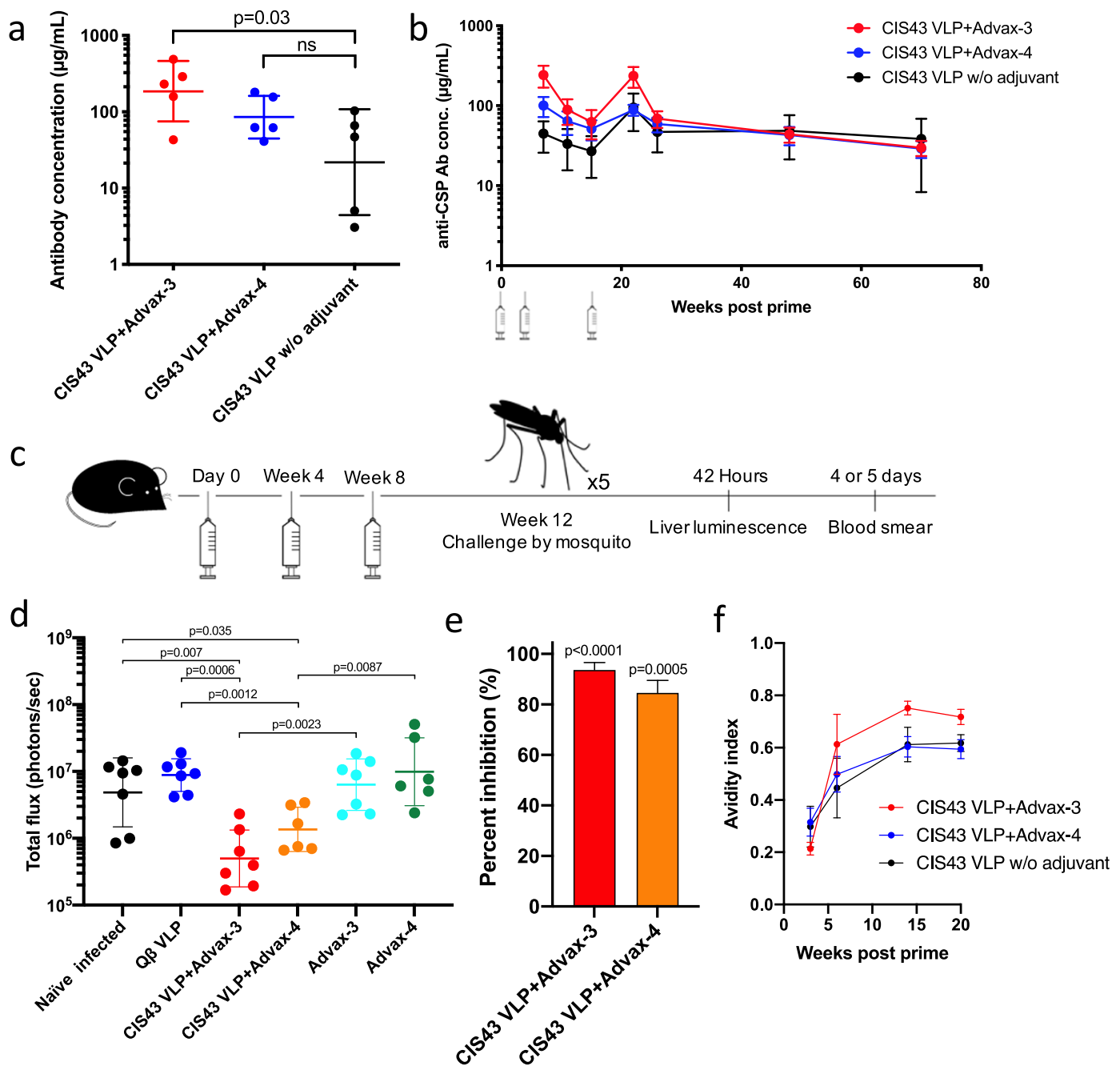

Fig. 4 Advax adjuvants enhance both CIS43 VLP immunogenicity and protection from malaria infection. a Anti-CSP antibody concentrations in Balb/c mice immunized two times with CIS43 VLPS with or without Advax-3 or Advax-4 ( $n=5 /$ group). Groups were statistically compared to one another by unpaired $t$-test. ns; not significant. b Kinetics of anti-CSP antibody concentrations in Balb/c mice immunized three times with CIS43 VLPs with or without adjuvant. Mice were immunized at week 0, 4, and 18, and anti-CSP antibody concentrations were measured at various timepoints following immunization. c Immunization and challenge scheme. Groups of C57BL/6 mice ( $n=6$ or $7 /$ group) were immunized three times and then challenged with five Pb-PfCSP-Luc infected mosquitos. Liver luminescence was evaluated $42 \mathrm{~h}$ following mosquito challenge. d Parasite liver load (as measured by luminescence) in CIS43 VLP-vaccinated or control mice following mosquito challenge. Mann-Whitney test was used to statistically compare each group to the unimmunized (naive) group. e Percent inhibition of liver infection, as calculated from luminescence data. Inhibition of infection is calculated relative to the mean signal in the negative control groups of mice. Mann-Whitney test was used to statistically compare each group to all control groups. Error bars represent SEM. f Avidity index (AI) of anti-CSP antibodies elicited following immunization with CIS43 VLPs with or without adjuvant. Error bars represent SEM. Statistical comparisons between groups was determined by unpaired $t$-test using area under the curve.

(Fig. 4b). Nevertheless, after this initial period of decline, anti-CSP IgG levels in groups immunized CIS43 VLPs plus Advax-3 or Advax4 were largely stable.

\section{Vaccination with CIS43 VLPs combined with Advax-3 or Advax-4 more strongly inhibits Plasmodium infection in mice} To evaluate the protection elicited by CIS43 VLPs adjuvanted with Advax-3 or Advax-4, C57BL/6 mice ( $n=6-7 /$ group) were vaccinated with CIS43 VLPs with adjuvant, or with adjuvant alone and then were challenged with Pb-PfCSP-Luc sporozoites. In this experiment, mice were challenged with live parasite-carrying mosquitoes to more closely recapitulate the conditions of natural infection (Fig. 4c). Mice immunized with CIS43 VLPs adjuvanted with Advax-3 or Advax-4 showed significantly lower parasite liver loads compared to the naive group, mice immunized with either adjuvant alone, or WT Q $\beta$ VLPs (Fig. 4d). Animals immunized with
CIS43 VLPs adjuvanted with Advax-3 or Advax-4 showed a $90 \%$ and a $72 \%$ reduction in liver parasite loads compared to naive mice, respectively. When we compared liver loads in these two groups with an aggregate of all of the negative control groups, we measured an 93\% (CIS43 VLPs with Advax-3) and a 84\% (CIS43 VLPs with Advax-4) reduction (Fig. 4e). Despite these reductions in liver load, all of the mice in this study developed parasitemia, as evaluated by blood smears, by day 4 post infection, with the exception of one mouse in each of the CIS43 VLP plus Advax groups-these mice, which had the lowest liver luminescence values in their respective groups, developed parasitemia at day 5 . These results demonstrate that the CIS43 VLP plus Advax vaccine is capable of stimulating high antibody responses that confers a robust, although not sterilizing, level of protection from liver infection in a mouse model.

It was previously reported in clinical trials of RTS,S/AS01 that increased antibody avidity, along with antibody concentration, 

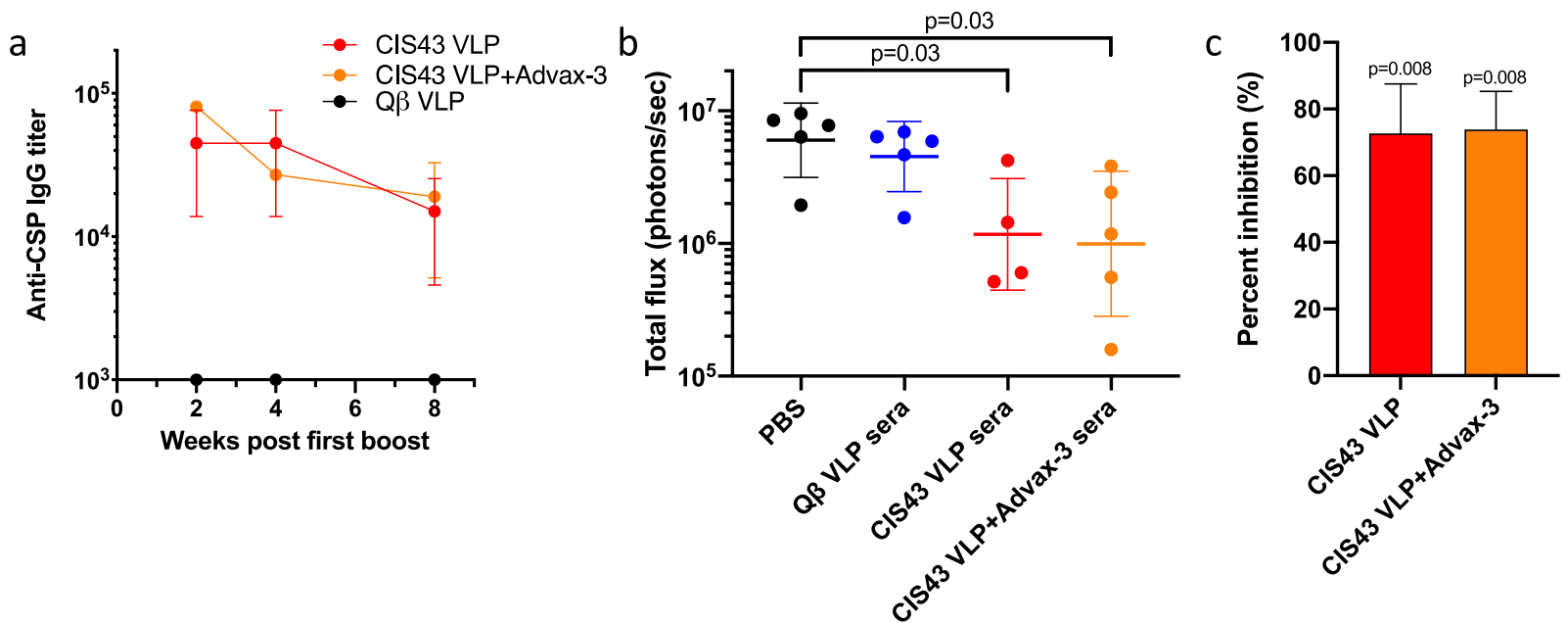

Fig. 5 Cynomolgus monkeys immunized with CIS43 VLPs produce protective anti-CSP antibodies. a IgG concentrations in cynomolgus monkeys immunized at weeks 0 and 4 with CIS43 VLPs with or without Advax-3 adjuvant ( $n=3 /$ group). Q $\beta$ VLPs were used as a negative control. b Parasite liver load (as measured by luminescence) in mice $(n=4-5 / g r o u p)$ that received an intravenous injection of $500 \mu l$ of sera from immunized cynomolgus monkeys. Two hours following serum transfer, mice were challenged with five Pb-PfCSP-Luc infected mosquitoes. Mann-Whitney test was used to statistically compare each group to the group that received an intravenous injection with PBS. c Percent inhibition of liver infection, as calculated from luminescence data. Inhibition of infection is calculated relative to the mean signal in the control groups. Mann-Whitney test was used to statistically compare each group to all control groups. Error bars represent SEM.

correlated with increased protection from clinical malaria ${ }^{19,35}$. To understand how avidity of CIS43 VLP-elicited antibody changes over time and in response to added adjuvant, we evaluated the avidity index (AI) of antibodies against CSP using a chaotropebased avidity assay. The mean Al of each group increased after each immunization, culminating in a high mean Al value $(>0.5)$ in all of the groups following the third immunization (Fig. 4f). Among the three vaccine groups we tested, Advax-3 adjuvanted CIS43 VLPs elicited anti-CSP antibodies with the highest avidity (Fig. 4f), although this difference was not statistically significant $(p=0.06)$.

\section{CIS43 VLPs elicit anti-CSP antibody responses in non-human primates and these antibodies can protect mice from malaria infection upon passive transfer}

CIS43 VLP immunogenicity in non-human primates was evaluated by immunizing groups $(n=3)$ of cynomolgus monkeys twice with unadjuvanted CIS43 VLPs or CIS43 VLPs adjuvanted with Advax-3. All of the macaques developed anti-CSP antibodies (Fig. 5a), but there was some heterogeneity in the responses, possibly due to the diverse ages and sizes of the animals in the study (Supplementary Table 1). Antibody levels were similar in macaques immunized with unadjuvanted CIS43 VLPs and mice immunized with CIS43 VLPs plus Advax-3. To investigate whether serum from CIS43 VLP-immunized cynomolgus monkeys could mediate protection from infection, we performed a passive transfer of monkey sera into naive C57BL/6 mice and then challenged the mice with Pb-PfCSP-Luc by mosquito infection. Macaque sera was obtained 2 weeks after the second immunization, pooled by immunization group, and then was used to passively immunize mice. Following intravenous administration of this pooled macaque sera, mice were rested for two hours and then subjected to mosquito challenge. As before, parasite liver load was evaluated by liver luminescence. We measured a statistically significant decrease in parasite liver burden in mice passively immunized with pooled sera from macaques immunized with unadjuvanted CIS43 VLP group and Advax-3-adjuvanted CIS43 VLPs, but not using sera from macaques immunized with control WT Q $\beta$ VLPs. Sera from monkeys immunized with unadjuvanted CIS43 VLPs reduced parasite liver load by $72 \%$, and sera from Advax-3-adjuvanted CIS43 VLPs reduced parasite liver load by $73 \%$. Thus, these data show that non-human primates immunized with CIS43 VLPs elicit antibody responses that are capable of blocking hepatocyte invasion.

\section{DISCUSSION}

Development of an effective vaccine for malaria has been complicated by a number of features of the parasite, including its complex life cycle, antigenic variability of its surface proteins, and the fact that immunity to natural infection largely does not confer protection from reinfection. One of the goals of an epitopebased malaria vaccine is to direct immune responses to target vulnerable, conserved epitopes from the pathogen. Here we evaluated the efficacy of a novel vaccine targeting a highly conserved epitope $14,34,36$ within the junctional region of $P$. falciparum CSP that is recognized by several potent inhibitory mAbs, including mAb CIS43. We showed that a VLP-based vaccine that displays the CIS43 epitope elicited high-titer antibodies against CSP in both mice and non-human primates and that vaccination inhibited parasite invasion in a mouse Plasmodium challenge model. Passive immunization of mice with CIS43immunized macaque sera also inhibited infection, indicating that antibodies mediate these prophylactic effects. The mechanism(s) by which antibodies induced by CIS43 VLPs provide protection is unclear, but it has been shown that the CIS43 mAb can partially inhibit proteolytic cleavage of $\mathrm{CSP}^{14}$, which is critical for invasion of hepatocytes ${ }^{37}$. CIS43 and the recently described mAb L9, an even more potent $m A b$ that recognizes an overlapping epitope in the junctional region, can also reduce hepatocyte infection in the mouse model by preventing parasite egress from sinusoids in the liver $^{15}$. CIS43, and other mAbs that target the junctional region of $\mathrm{CSP}^{16}$, also cross-reacts with the central repeat region of CSP. Antibodies that bind to the central repeat region of CSP can block infection by inhibiting sporozoite motility ${ }^{18,34,38}$, inducing parasite cytotoxicity $^{39}$ and/or by enhancing immune clearance ${ }^{40,41}$. We showed that antibodies induced by CIS43 VLPs bound most strongly to the CIS43 epitope, but they also cross-reacted with other CSP epitopes in the junctional region and the central repeat region, raising the possibility that this binding promiscuity may 
contribute to their inhibitory activity. Thus, it is possible that polyreactive antibodies targeting the junctional region of CSP act through multiple mechanisms to prevent hepatocyte infection. Additional studies will be required to provide insight into the major mechanisms whereby vaccines targeting the CIS43 epitope inhibit hepatocyte invasion. Producing a vaccine that specifically targets the R1 cleavage site in the junctional region could potentially dissect the relative contributions of how antibody binding to these two regions contributes to protection.

The junctional region at the $\mathrm{N}$ terminus of the central repeat region of CSP contains an NPDP tetrapeptide followed by three interspersed NANP and NVDP repeats (Supplementary Fig. 1). This is followed by the central repeat region, which consists of $\sim 35-38$ NANP repeats, with a fourth NVDP tetrapeptide typically inserted after the twentieth NANP repeat. Several studies have shown that inhibitory CSP mAbs recognize epitopes derived from the joining of major and/or minor tetrapeptide repeats, including the sequences NPNA (major/major), NPNV (major/minor), and DPNA (minor/major) ${ }^{15,42,43}$. CIS43, e.g., strongly binds to DPNAcontaining sequences from $\mathrm{CSP}^{15}$. In contrast, $\mathrm{L9}$, which is likely the most potent anti-CSP mAb described to date ( 4-fold more potent than CIS43), prefers binding to the NPNV motifs that form at the junctions of interspersed NANP and NVDP repeats ${ }^{15}$. The CIS43 epitope-derived peptide that we utilized in this vaccine study contains two DPNA motifs and a single NPNV motif, and antibodies raised by immunization with CIS43 VLPs bound most strongly to peptides containing DPNA motifs (Fig. 2c). Given the potency of the L9 $\mathrm{mAb}$, it would be interesting to test whether a VLP-based vaccine that preferentially induced antibodies targeting the NPNV motif could induce more strongly protective antibody responses. Such a vaccine could potentially be combined with the CIS43 epitope-targeted vaccine described in this study.

The ability to elicit durable antibody responses through the induction of long-lived antibody secreting plasma cells is likely to be particularly important for providing protection from malaria infection. Liver infection occurs within a few minutes or hours of parasite exposure and once Plasmodium invades hepatocytes it is not accessible to anti-sporozoite antibodies ${ }^{44}$. Thus, circulating antibodies, but not reactivated memory B cells, are likely to be a critical mediator of effective immunity to malaria sporozoites. Indeed, one of the primary shortcomings of RTS,S is that antibody levels decrease rapidly following immunization ${ }^{45}$. Here we showed that CIS43 VLPs not only elicited high titer antibody responses against CSP, but that these antibodies were extremely durable. Over the span of a nearly 2-year period following vaccination, mouse anti-CSP antibody titers did not decline. These data add to a growing body of literature demonstrating that the ability to induce LLPCs is a feature of vaccine antigens, such as VLPs, with highly multivalent structures ${ }^{29-31}$. Our study provides evidence that use of a multivalent vaccine technology can effectively elicit durable antibody responses against CSP.

There have been several other efforts to develop multivalent vaccines targeting CSP. R21 is a RTS,S-like vaccine in which the central repeat region of CSP is displayed at much higher density on recombinant HBsAg particles than RTS,S. R21 elicits potent immune responses against $\operatorname{CSP}^{46}$ and is currently in Phase $1 / 2 \mathrm{a}$ clinical trials. Several groups have applied a similar approach using bacteriophage VLP-based vaccines to display full-length CSP at high valency and have shown that these vaccines are immunogenic in mice ${ }^{47}$ and can confer protection following transgenic parasite challenge ${ }^{48}$. Whitacre et al. ${ }^{27}$ developed epitope-targeted vaccines that employ woodchuck hepatitis virus core antigen (WHcAg) VLPs to display B- and T-cell epitopes from repeat and non-repeat regions of CSP. VLPs displaying repeat, but not nonrepeat, epitopes were capable of eliciting sterilizing immunity that prevented blood-stage infection in a mouse model. These latter challenge studies utilized a transgenic $\mathrm{Pb}$ CSP that only contained the repeat region of PFCSP (unlike our studies in which transgenic
$P b$ carried full-length PfCSP). Thus, it is difficult to compare the relative protection provided by WHcAg-based VLPs versus the CIS43 VLPs described in this study. Nevertheless, a common finding is that VLP display of CSP epitopes by multiple platform technologies is effective at eliciting high-titer antibody responses against CSP.

We have previously shown that bacteriophage VLPs can induce strong antibody responses in the absence of exogenous adjuvants; co-administration with most standard adjuvant formulations only resulted in modest boosts in antibody titer ${ }^{49}$. This is due to the repetitive structure of VLPs, which strongly stimulates B cells ${ }^{21,50}$, but also the fact that bacteriophage VLPs encapsidate single stranded RNA, which can serve as a natural endogenous adjuvant. Here we showed that combining CIS43 VLPs with two different combination adjuvants, one combining a TLR9-active oligonucleotide (CpG55.2) with aluminum hydroxide (Advax-3) and the other combining delta inulin with a TLR7/8 agonist (Advax-4) substantially increased immunogenicity in mice relative to unadjuvanted CIS43 VLPs, although the addition of adjuvant did not boost antibody titers in non-human primates. Importantly, Advax adjuvants have been shown to be safe and efficacious in multiple human vaccine trials ${ }^{26}$ and recently the US Food and Drug Administration approved an IND application for an enhanced seasonal influenza vaccine containing an Advax-CpG formulation. Although the use of Advax adjuvants increased peak antibody concentrations, one limitation is that these levels rapidly declined to concentrations that were similar to the levels observed in mice immunized with unadjuvanted CIS43 VLPs.

Although the monoclonal-like response conferred by the single epitope displaying CIS43 VLPs did not confer sterilizing immunity against malaria in a mouse challenge model, the degree of protection elicited by this vaccine highlights the importance of this particular epitope for future clinical applications, and the potential efficacy of additional VLP-based vaccines that target critical epitopes within the junctional region of CSP. Further development of CIS43 VLPs, in combination with vaccines that induce antibody and/or T-cell responses against other liver- or blood-stage malarial antigens, could increase the breadth and potency of protection.

\section{METHODS}

\section{Production and characterization of CIS43 epitope displaying} bacteriophage $\mathbf{Q} \boldsymbol{\beta}$ VLPs

$\mathrm{Q} \beta$ bacteriophage VLPs were produced in Escherichia coli using methods previously described for the production of bacteriophage PP7 VLPs ${ }^{51}$. With the exception of the VLPs used in preliminary mouse immunogenicity studies, all CIS43 VLPs and WT Q $\beta$ VLPs were depleted of endotoxin by three rounds of phase separation using Triton X-114 (Sigma-Aldrich), as described in ref. ${ }^{52}$. The fifteen amino acid CIS43 epitope peptide was synthesized (GenScript) and modified to contain a C-terminal gly-gly-glycys linker sequence (NPDPNANPNVDPNANGGGC). The peptide was conjugated directly to surface lysines on $Q \beta$ bacteriophage VLPs using the bidirectional crosslinker succinimidyl 6-[( $\beta$-maleimidopropionamido) hexanoate] (SMPH; Thermo Fisher Scientific) as previously described ${ }^{51}$. The efficiency of conjugation was assessed by gel electrophoresis using a $10 \%$ SDS denaturing polyacrylamide gel. Peptide conjugation results in a mobility shift of the $\mathrm{Q} \beta$ coat protein due to an increase in molecular weight. The percentage of coat protein with zero, one, two, or more attached peptides was determined using ImageJ software and used to calculate average peptide density per VLP. Presence of the CIS43 peptide on CIS43 VLPS was confirmed by ELISA. Briefly, $250 \mathrm{ng}$ of VLPs were used to coat wells of an ELISA plate. Wells were probed with dilutions of $\mathrm{mAb}$ CIS43 (generously provided by Robert Seder, NIH Vaccine Research Center), followed by a 1:4000 dilution of horseradish peroxidase (HRP) labeled goat anti-human IgG (Jackson Immunoresearch). The reaction was developed using 3,3',5,5'-tetramethylbenzidine (TMB) substrate (Thermo Fisher Scientific) and stopped using $1 \% \mathrm{HCl}$. Reactivity of the CIS43 mAb for the CIS43 VLPs was determined by measuring optical density at $450 \mathrm{~nm}$ $\left(\mathrm{OD}_{450}\right)$ using an AccuSkan plate reader (Fisher Scientific). For TEM analysis, 
VLPs were coated onto carbon support film on copper grids and stained with $1 \%$ uranyl acetate for 1 min. VLPs were imaged with Hitachi HT7700 TEM with an AMT XR16M digital camera.

\section{Ethics statement for animal studies}

All animal research complied with the Institutional Animal Care and Use Committee of the University of New Mexico School of Medicine (Approved protocol number: 19-200870-HSC), Johns Hopkins University (Approved protocol permit number: MO18H419). AlphaGenesis, Inc. adheres to the $\mathrm{NIH}$ Office of Laboratory Animal Welfare standards (OLAW welfare assurance \#A3645-01) and all guidelines of AGI Ethics and Compliance Program were followed.

\section{Mouse immunization studies}

Groups of 4-week-old female Balb/c mice (obtained from the Jackson Laboratory) were immunized intramuscularly with $5 \mu \mathrm{g}$ of CIS43 VLPs or control (unmodified) Q $\beta$ VLPs. Mice were typically boosted twice after the initial prime, at 3- or 4-week intervals. Some vaccinations were performed using proprietary adjuvants generously provided by Vaxine Ptd Ltd. These adjuvants include Advax-1 (delta inulin alone), Advax-2 (delta inulin plus a TLR9 agonist), Advax-3 (CpG55.2 [a TLR9 agonist described below] plus aluminum hydroxide), Advax-4 (delta inulin plus a TLR7/8 agonist), and Advax-5 (delta inulin plus a TLR4 agonist). In these experiments, mice were intramuscularly immunized with $5 \mu \mathrm{g}$ VLPs in combination with $20 \mu \mathrm{l}$ the following: Advax $(50 \mathrm{mg} / \mathrm{mL})$, Advax-2 $(50 \mathrm{mg} / \mathrm{mL})$, Advax-3 $(5 \mathrm{mg} / \mathrm{mL})$, Advax-4 (50 mg/mL), Advax-5 (50 mg/mL), and CpG55.2 (0.5 mg/mL)—a synthetic Class B CpG oligonucleotide adjuvant that is dually active on both mouse and human TLR9 (unpublished data). An additional group of mice received $5 \mu \mathrm{g}$ of unadjuvanted CIS43 VLPs. In these experiments, mice were boosted four weeks after the prime and selected groups received a second boost at 3 months. In each mouse experiment, serum samples were collected prior to each boost and, in some cases, at additional later timepoints following the final boost.

\section{Cynomolgus monkey immunization studies}

Groups ( $n=3$ /group) of male and female cynomolgus monkeys (Macaca fascicularis) of varying ages (9.9-17 years) and body weights $(4.00-13.30 \mathrm{~kg})$ were immunized with $100 \mu \mathrm{g}$ of unadjuvanted CIS43 VLPs, or the same dose in combination with $0.5 \mathrm{mg}$ of Advax-3. One month after the prime, animals were boosted with $50 \mu \mathrm{g}$ of VLPs with or without Advax-3. A negative control group received similar doses of unmodified WT Q $\beta$ VLPs. Serum was collected at the initial immunization and at 2week intervals thereafter.

\section{Quantitating antibody responses}

Serum antibodies against full-length CSP were detected by ELISA using recombinant CSP expressed in Pseudomonas fluorescens ${ }^{53}$ (and generously provided by Gabriel Gutierrez at Leidos, Inc.) as the coating antigen. Immulon 2 plates (Thermo Scientific) were coated with $250 \mathrm{ng}$ of CSP in $50 \mu \mathrm{l}$ phosphate-buffered saline (PBS) and incubated at $4{ }^{\circ} \mathrm{C}$ overnight. Following incubation, wells were blocked with PBS- $0.5 \%$ milk for $2 \mathrm{~h}$ at room temperature. Sera isolated from immunized animals were serially diluted in PBS- $0.5 \%$ milk and applied to wells and incubated at room temperature for $2.5 \mathrm{~h}$. Reactivity to the target antigen was detected using HRP-labeled goat anti-mouse IgG or, for macaque sera, HRP-labeled goat anti-human IgG (both Jackson Immunoresearch, diluted $1: 4000$ ). Reactivity was determined using TMB substrate as described above. End-point dilution titer was defined as the greatest sera dilution that yielded an $\mathrm{OD}_{450}>2$-fold over background. For mouse sera, anti-CSP antibody concentrations were also quantitated by ELISA by generating a standard curve using known concentrations of the anti-CSP mouse mAb $2 A 10^{7,31,48}$. For isotype analysis, mouse serum samples were diluted $1: 10,000$, and isotype-specific responses were determined using HRP-labeled rat antimouse lgG1 or lgG2a (Zymed, diluted 1:4000).

For peptide ELISAs, Immulon 2 plates were coated with $500 \mathrm{ng}$ streptavidin (Invitrogen) for $2 \mathrm{~h}$ at $37^{\circ} \mathrm{C}$. Following washing, SMPH was added to wells at $1 \mu \mathrm{g} / \mathrm{well}$ and incubated for $1 \mathrm{~h}$ at room temperature. Specific peptides were added to the wells at $1 \mu \mathrm{g} / \mathrm{well}$ and incubated overnight at $4{ }^{\circ} \mathrm{C}$. Wells were then incubated with dilutions of mouse sera and binding was detected as described above.

Competition ELISAs were performed by using the CSP ELISA protocol, as described above, with the following modifications: after mouse serum was added to the plate, $40 \mathrm{ng}$ of the human $\mathrm{mAb}$ CIS43 (at a final concentration of $400 \mathrm{ng} / \mathrm{mL}$ ) was added to each well and incubated for $30 \mathrm{~min}$. CIS43 mAb binding to CSP was detected using HRP-labeled goat anti-human IgG at a $1: 4000$ dilution.

\section{Measurement of antibody avidity}

The Al of anti-CSP antibodies was evaluated using an ELISA-based chaotrope avidity assay ${ }^{54}$. This protocol followed the standard ELISA (described above), except that following serum incubation wells were treated with $6 \mathrm{M}$ urea for $10 \mathrm{~min}$. Serum dilutions corresponding to comparable $O D$ value for each group were chosen to control for differences in antibody concentrations. The Al was calculated as the ratio of the ELISA absorbance value of $6 \mathrm{M}$ urea-treated wells $\left(A_{6 M}\right.$ UREA $)$ to control wells incubated in water $\left(A_{c}\right) ; \mathrm{Al}=A_{6 \mathrm{M} \text { UREA }} / A_{c}$. Multiple dilutions of sera were analyzed and all samples were tested in duplicate.

\section{Mouse Pb-PfCSP-Luc sporozoite challenge}

Challenge studies were performed using female 6-8-week-old C57BL/6 mice. Mice (typically $n=7 /$ group) were immunized intramuscularly with $5 \mu \mathrm{g}$ of CIS43 VLPs with or without adjuvant three times at 3-week intervals. Separate control groups were immunized with unmodified WT $\mathrm{Q} \beta$ VLPs, PBS, or adjuvant alone. Each immunogen was blinded to minimize the potential for bias in animal handling during the challenge portion of the study. Serum was collected following the third immunization.

Mice were challenged using transgenic $P$. berghei sporozoites engineered to express luciferase and full-length $P$. falciparum CSP in place of $P$. berghei CSP (denoted as Pb-PfCSP-Luc) ${ }^{32}$. Mice were challenged directly using sporozoites or by using infected mosquitos. For the sporozoite challenge, Pb-PfCSP-Luc sporozoites were freshly harvested from female Anopheles stephensi salivary glands. One thousand sporozoites in $200 \mu \mathrm{l}$ Hank's buffered salt solution $/ 2 \%$ fetal calf serum were intravenously injected into immunized and naive mice. Forty-two hours post challenge, mice were intraperitoneally injected with $100 \mu \mathrm{l}$-luciferin $(30 \mathrm{mg} / \mathrm{ml})$ and anesthetized. Liver luminescence was assessed by IVIS Spectrum Imaging System (Perkin Elmer). For mosquito challenges, A. stephensi mosquitos were infected by blood feeding on Pb-PfCSP-Luc infected mice. Prior to challenge, mice were anesthetized with $2 \%$ Avertin and then exposed to five mosquitos for a blood meal. Following feeding, mosquitos positive for a blood meal were counted. Liver luminescence was assessed $42 \mathrm{~h}$ post challenge, as described above. Five days later, blood smears were evaluated by Giemsa staining for parasitemia.

\section{Passive transfer study}

Cynomolgus monkey sera was pooled within each group, heat inactivated for $30 \mathrm{~min}$ at $56^{\circ} \mathrm{C}$, and filtered through a 0.45 micron filter to remove aggregates. Sera ( $500 \mu \mathrm{l}$; or PBS as a control) was then passively transferred into each mouse ( $n=4-5$ mice/group) intravenously via the tail vein by slow injection. Two hours following serum transfer, mice were challenged by $A$. stephensi mosquito bite, as described above. Liver luminescence was evaluated $42 \mathrm{~h}$ post challenge, and parasitemia was evaluated by blood smears 4 days later, as described above.

\section{Statistics}

All statistical analyses of data were performed using GraphPad Prism 8 using two-sided tests. For percent inhibition calculations (Figs. 3c, 4e, and 5c), the liver luminescence values of individual vaccinate mice was divided by the mean of mice in negative control groups. Background luminescence levels $\left(10^{5}\right)$ were subtracted from all values.

\section{Reporting summary}

Further information on research design is available in the Nature Research Reporting Summary linked to this article.

\section{DATA AVAILABILITY}

The datasets used and/or analyzed in the current study are available from the corresponding author upon reasonable request. 
Received: 7 August 2020; Accepted: 11 December 2020; Published online: 21 January 2021

\section{REFERENCES}

1. WHO. World Malaria Report 2019 (WHO, 2019).

2. Beeson, J. G. et al. Challenges and strategies for developing efficacious and longlasting malaria vaccines. Sci. Transl. Med. 11, eaau1458 (2019).

3. Nussenzweig, V. \& Nussenzweig, R. S. Rationale for the development of an engineered sporozoite malaria vaccine. Adv. Immunol. 45, 283-334 (1989).

4. Cohen, J., Nussenzweig, V., Nussenzweig, R., Vekemans, J. \& Leach, A. From the circumsporozoite protein to the RTS, S/AS candidate vaccine. Hum. Vaccin. 6 , 90-96 (2010)

5. Zhao, J., Bhanot, P., Hu, J. \& Wang, Q. A comprehensive analysis of Plasmodium circumsporozoite protein binding to hepatocytes. PLOS ONE 11, 1-13 (2016).

6. Hollingdale, M. R., Nardin, E. H., Tharavanij, S., Schwartz, A. L. \& Nussenzweig, R. S. Inhibition of entry of Plasmodium falciparum and $P$. vivax sporozoites into cultured cells; an in vitro assay of protective antibodies. J. Immunol. 132, 909-913 (1984).

7. Zavala, F. et al. Rationale for development of a synthetic vaccine against Plasmodium falciparum malaria. Science 228, 1436-1440 (1985).

8. Dame, J. B. et al. Structure of the gene encoding the immunodominant surface antigen on the sporozoite of the human malaria parasite Plasmodium falciparum. Science 225, 593-599 (1984).

9. Stoute, J. A. et al. A Preliminary evaluation of a recombinant circumsporozoite protein vaccine against Plasmodium falciparum malaria. N. Engl. J. Med. 336 86-91 (1997).

10. Moorthy, V. S. \& Ballou, W. R. Immunological mechanisms underlying protection mediated by RTS,S: a review of the available data. Malar. J. 8, 1-7 (2009).

11. Penny, M. A., Pemberton-Ross, P. \& Smith, T. A. The time-course of protection of the RTS,S vaccine against malaria infections and clinical disease. Malar. J. 14, 1-13 (2015).

12. Olotu, A. et al. Seven-year efficacy of RTS, S/AS01 malaria vaccine among young african children. N. Engl. J. Med. 374, 2519-2529 (2016).

13. Tan, J. et al. A public antibody lineage that potently inhibits malaria infection by dual binding to the circumsporozoite protein. Nat. Med. 24, 401-407 (2018).

14. Kisalu, N. K. et al. A human monoclonal antibody prevents malaria infection by targeting a new site of vulnerability on the parasite. Nat. Med. 24, 408-416 (2018).

15. Wang, L. T. et al. A potent anti-malarial human monoclonal antibody targets circumsporozoite protein minor repeats and neutralizes sporozoites in the liver. Immunity 53, 733-744.e8 (2020).

16. Oyen, D. et al. Structure and mechanism of monoclonal antibody binding to the junctional epitope of Plasmodium falciparum circumsporozoite protein. PLoS Pathog. 16, 1-22 (2020).

17. NIAID. Trial to Evaluate CIS43LS in Healthy Adults. https://clinicaltrials.gov/ct2/ show/NCT04206332 (2020).

18. Flores-Garcia, Y. et al. Antibody-mediated protection against plasmodium sporozoites begins at the dermal inoculation site. MBio 9, 1-14 (2018).

19. Dobaño, C. et al. Concentration and avidity of antibodies to different circumsporozoite epitopes correlate with RTS,S/AS01 malaria vaccine efficacy. Nat Commun. 10, 1-13 (2019).

20. Chackerian, B. Virus-like particles: flexible platforms for vaccine development. Expert Rev. Vaccines 6, 381-390 (2007).

21. Frietze, K. M., Peabody, D. S. \& Chackerian, B. Engineering virus-like particles as vaccine platforms. Curr. Opin. Virol. 18, 44-49 (2016)

22. Chackerian, B. \& Frietze, K. M. Moving towards a new class of vaccines for noninfectious chronic diseases. Expert Rev. Vaccines 15, 561-563 (2016)

23. Mata, E., Salvador, A., Igartua, M., Hernández, R. M. \& Pedraz, J. L. Malaria vaccine adjuvants: latest update and challenges in preclinical and clinical research Biomed. Res. Int. 2013, 282913 (2013).

24. Roestenberg, M. et al. Safety and immunogenicity of a recombinant Plasmodium falciparum AMA1 malaria vaccine adjuvanted with Alhydrogel, Montanide ISA 720 or AS02. PLOS ONE 3, e3960 (2008).

25. Honda-Okubo, Y., Saade, F. \& Petrovsky, N. AdvaxTM, a polysaccharide adjuvant derived from delta inulin, provides improved influenza vaccine protection through broad-based enhancement of adaptive immune responses. Vaccine $\mathbf{3 0}$, 5373-5381 (2012).

26. Petrovsky, N. \& Cooper, P. D. AdvaxTM, a novel microcrystalline polysaccharide particle engineered from delta inulin, provides robust adjuvant potency together with tolerability and safety. Vaccine 33, 5920-5926 (2015).

27. Whitacre, D. C. et al. P. falciparum and P. vivax epitope-focused VLPs elicit sterile immunity to blood stage infections. PLOS ONE 10, 1-22 (2015).
28. Espinosa, D. A. et al. Proteolytic cleavage of the Plasmodium falciparum circumsporozoite protein is a target of protective antibodies. J. Infect. Dis. 212, 1111-1119 (2015).

29. Safaeian, M. et al. Durable antibody responses following one dose of the bivalent human papillomavirus L1 virus-like particle vaccine in the Costa Rica vaccine trial. Cancer Prev. Res. 6, 1242-1250 (2013).

30. Amanna, I. J., Carlson, N. E. \& Slifka, M. K. Duration of humoral immunity to common viral and vaccine antigens. N. Engl. J. Med. 357, 1903-1905 (2007).

31. Chackerian, B. \& Peabody, D. S. Factors that govern the induction of long-lived antibody responses. Viruses 12, 74 (2020).

32. Flores-Garcia, Y. et al. Optimization of an in vivo model to study immunity to Plasmodium falciparum pre-erythrocytic stages. Malar. J. 18, 1-9 (2019).

33. Santos, B. et al. Advax4 delta inulin combination adjuvant together with ECMX, a fusion construct of four protective $\mathrm{MTB}$ antigens, induces a potent Th1 immune response and protects mice against Mycobacterium tuberculosis infection. Hum Vaccines Immunother. 13, 2967-2976 (2017).

34. Nardin, E. H. et al. Circumsporozoite proteins of human malaria parasites Plasmodium falciparum and Plasmodium vivax. J. Exp. Med. 156, 20-30 (1982).

35. Ajua, A. et al. The effect of immunization schedule with the malaria vaccine candidate RTS,S/AS01E on protective efficacy and anti-circumsporozoite protein antibody avidity in African infants. Malar. J. 14, 1-6 (2015).

36. Rich, S. M., Hudson, R. R. \& Ayala, F. J. Plasmodium falciparum antigenic diversity evidence of clonal population structure. Proc. Natl Acad. Sci. USA 94 13040-13045 (1997).

37. Coppi, A. et al. The malaria circumsporozoite protein has two functional domains, each with distinct roles as sporozoites journey from mosquito to mammalian host. J. Exp. Med. 208, 341-356 (2011).

38. Vanderberg, J. \& Frevert, U. Intravital microscopy demonstrating antibodymediated immobilisation of Plasmodium berghei sporozoites injected into skin by mosquitoes. Int. J. Parasitol. 34, 991-996 (2004).

39. Aliprandini, E. et al. Cytotoxic anti-circumsporozoite antibodies target malaria sporozoites in the host skin. Nat. Microbiol. 3, 1224-1233 (2018).

40. Kurtovic, L. et al. Human antibodies activate complement against Plasmodium falciparum sporozoites, and are associated with protection against malaria in children. BMC Med. 16, 1-17 (2018).

41. Schwenk, R. et al. Opsonization by antigen-specific antibodies as a mechanism of protective immunity induced by Plasmodium falciparum circumsporozoite protein-based vaccine. Parasite Immunol. 25, 17-25 (2003).

42. Oyen, D. et al. Structural basis for antibody recognition of the NANP repeats in Plasmodium falciparum circumsporozoite protein. Proc. Natl Acad. Sci. USA 114 E10438-E10445 (2017).

43. Ghasparian, A., Moehle, K., Linden, A. \& Robinson, J. A. Crystal structure of an NPNA-repeat motif from the circumsporozoite protein of the malaria parasite Plasmodium falciparum. Chem. Commun. 3, 174-176 (2006).

44. Sinnis, P. \& Zavala, F. The skin: where malaria infection and the host immune response begin. Semin. Immunopathol. 34, 787-792 (2012).

45. Kurtovic, L. et al. Induction and decay of functional complement-fixing antibodies by the RTS,S malaria vaccine in children, and a negative impact of malaria exposure. BMC Med. 17, 1-14 (2019).

46. Collins, K. A., Snaith, R., Cottingham, M. G., Gilbert, S. C. \& Hill, A. V. S. Enhancing protective immunity to malaria with a highly immunogenic virus-like particle vaccine. Sci. Rep. 7, 1-15 (2017).

47. Janitzek, C. M. et al. Bacterial superglue generates a full-length circumsporozoite protein virus-like particle vaccine capable of inducing high and durable antibody responses. Malar. J. 15, 1-9 (2016).

48. Khan, F. et al. Head-to-head comparison of soluble vs. Q $\beta$ VLP circumsporozoite protein vaccines reveals selective enhancement of NANP repeat responses. PLoS ONE 10, 1-18 (2015).

49. Tumban, E., Peabody, J., Peabody, D. S. \& Chackerian, B. A universal virus-like particle-based vaccine for human papillomavirus: Longevity of protection and role of endogenous and exogenous adjuvants. Vaccine 31, 4647-4654 (2013).

50. Irvine, D. J. \& Read, B. J. Shaping humoral immunity to vaccines through antigendisplaying nanoparticles. Curr. Opin. Immunol. 65, 1-6 (2020).

51. Tumban, E., Peabody, J., Peabody, D. S. \& Chackerian, B. A Pan-HPV vaccine based on bacteriophage PP7 VLPs displaying broadly cross-neutralizing epitopes from the HPV minor capsid protein, L2. PLoS ONE 6, 1-11 (2011).

52. Aida, Y. \& Pabst, M. Removal of endotoxin from protein solutions by phase separation using triton X-114. J. Immunol. Methods 132, 191-195 (1990).

53. Noe, A. R. et al. A full-length Plasmodium falciparum recombinant circumsporozoite protein expressed by Pseudomonas fluorescens platform as a malaria vaccine candidate. PLOS ONE 9, e107764 (2014).

54. Fialová, L., Petráčková, M. \& Kuchař, O. Comparison of different enzyme-linked immunosorbent assay methods for avidity determination of antiphospholipid antibodies. J. Clin. Lab. Anal. 31, 1-9 (2017). 


\section{ACKNOWLEDGEMENTS}

This study was supported, in part, by a generous contribution to the UNM Foundation in honor of Jeffrey Michael Gorvetzian in support of biomedical research excellence at the University of New Mexico School of Medicine and by funding from National Institute of Allergy and Infectious Diseases of the National Institutes of Health grant T32-A1007538. F.Z., H.J., and A.E. thank the Bloomberg Philanthropies for continued support. Development of Advax adjuvants was supported by funding from National Institute of Allergy and Infectious Diseases of the National Institutes of Health under Contracts HHS-N272201400053C, HHS-N272200800039C and U01-AI061142.

\section{AUTHOR CONTRIBUTIONS}

L.J., F.Z., N.P., and B.C. designed the study and drafted the manuscript. L.J. engineered the VLPs, performed the mouse experiments at the University of New Mexico, performed the analysis of immune responses, and analyzed all data. H.J., A.E., and F.Z. performed the mouse challenge experiments and statistically analyzed the data resulting from those experiments. N.P. supplied adjuvants and coordinated the macaque immunizations.

\section{COMPETING INTERESTS}

B.C. has equity stakes in Agilvax, Inc. and FL72, companies that do not have financial interest in malaria vaccines. N.P. is affiliated with Vaxine Pty Ltd, a company having a financial interest in Advax adjuvants. The other authors declare that they have no known competing financial interests or personal relationships that could have appeared to influence the work reported in this paper.

\section{ADDITIONAL INFORMATION}

Supplementary information is available for this paper at https://doi.org/10.1038/ s41541-020-00274-4.

Correspondence and requests for materials should be addressed to B.C.

Reprints and permission information is available at http://www.nature.com/ reprints

Publisher's note Springer Nature remains neutral with regard to jurisdictional claims in published maps and institutional affiliations.

Open Access This article is licensed under a Creative Commons Attribution 4.0 International License, which permits use, sharing, adaptation, distribution and reproduction in any medium or format, as long as you give appropriate credit to the original author(s) and the source, provide a link to the Creative Commons license, and indicate if changes were made. The images or other third party material in this article are included in the article's Creative Commons license, unless indicated otherwise in a credit line to the material. If material is not included in the article's Creative Commons license and your intended use is not permitted by statutory regulation or exceeds the permitted use, you will need to obtain permission directly from the copyright holder. To view a copy of this license, visit http://creativecommons. org/licenses/by/4.0/.

(c) The Author(s) 2021 